\title{
Obesidade na adolescência: um problema de Saúde Pública
}

\author{
Teen obesity: a Public Health problem
}

Obesidad adolescente: un problema de Salud Pública

\begin{abstract}
Lais Vanessa dos Santos Guimarães ${ }^{1}$, Mariane Araújo Ramos ${ }^{1}$, Maurício José Cordeiro Souza ${ }^{1}$, Dirley Cardoso Moreira², José Luiz Picanço da Silva², Rubens Alex de Oliveira Menezes²*.
\end{abstract}

\section{RESUMO}

Objetivo: Analisar evidências científicas relacionadas à obesidade na adolescência. E como objetivos específicos: identificar os principais fatores relacionados com a obesidade e identificar os benefícios dos cuidados de enfermagem na prevenção da obesidade na adolescência. Métodos: Trata-se de uma Revisão Integrativa da Literatura para análise da produção do conhecimento científico sobre o tema investigado, a presente pesquisa será do tipo de caráter qualitativo. Utilizados como filtro a base de dados: MEDLINE, LILACS e BDENF. Resultados: Nesta revisão foram analisados 07 artigos que atenderam aos critérios de inclusão previamente estabelecidos. Os artigos abordam sobre os hábitos alimentares não saudáveis com estilo de vida inadequado, associada aos fatores genéticos e ambientais, que influenciam na vida de adolescentes sedentários com obesidade. Adicionalmente, os artigos enfatizam a problemática da obesidade na adolescência, tornando-se um problema de saúde pública, relacionada a vários riscos de doenças associadas, como diabetes e doenças cardiovasculares (DCV), bem como, neoplasias, problemas respiratórios, dermatológicos e ortopédicos decorrentes de fatores genéticos, ambientais e de estilo de vida inadequado. Considerações finais: A obesidade na adolescência, é um grande problema de saúde pública, sendo importante o conhecimento da história natural da doença e das comorbidades para permitir o diagnóstico precoce e o tratamento destas condições.

Palavras-chave: Enfermagem, Adolescente, Obesidade.

\begin{abstract}
Objective: To analyze scientific evidence related to obesity in adolescence. And as specific objectives: to identify the main factors related to obesity and to identify the benefits of nursing care in preventing obesity in adolescence. Methods: This is an Integrative Literature Review to analyze the production of scientific knowledge on the subject investigated the present research will be of a qualitative nature. The database was used as a filter: MEDLINE, LILACS and BDENF. Results: In this review, 07 articles were analyzed that met the inclusion criteria previously established. The articles address unhealthy eating habits with an inadequate lifestyle, associated with genetic and environmental factors, which influence the lives of sedentary adolescents with obesity. Additionally, the articles emphasize the problem of obesity in adolescence, becoming a public health problem, related to several risks of associated diseases, such as diabetes and cardiovascular diseases (CVD), as well as neoplasms, respiratory, dermatological, and orthopedic problems resulting genetic, environmental, and inappropriate lifestyle factors. Final considerations: Obesity in adolescence is a major public health problem, and it is important to know the natural history of the disease and comorbidities to allow early diagnosis and treatment of these conditions.
\end{abstract}

Keywords: Nursing, Adolescent, Obesity.

\section{RESUMEN}

Objetivo: Analizar la evidencia científica relacionada con la obesidad en la adolescencia. Y como objetivos específicos: identificar los principales factores relacionados con la obesidad e identificar los beneficios del cuidado de enfermería en la prevención de la obesidad en la adolescencia. Métodos: Se trata de una Revisión de Literatura Integrativa para analizar la producción de conocimiento científico sobre el tema investigado, la presente investigación será de carácter cualitativo. Se utilizó como filtro la base de datos: MEDLINE, LILACS y BDENF. Resultados: En esta revisión se analizaron 07 artículos que cumplieron con los criterios de inclusión previamente establecidos. Los artículos abordan hábitos alimentarios poco saludables con un estilo de vida inadecuado, asociados a factores genéticos y ambientales, que influyen en la vida de los adolescentes sedentarios con obesidad. Adicionalmente, los artículos enfatizan el problema de la obesidad en la adolescencia, convirtiéndose en un problema de salud pública, relacionado con varios riesgos de enfermedades asociadas, como diabetes y

\footnotetext{
${ }^{1}$ Faculdade Madre Tereza (FAMAT), Santana - AP.

${ }^{2}$ Universidade Federal do Amapá (UNIFAP), Macapá - AP. *E-mail: rubens.alex@unifap.br
} 
enfermedades cardiovasculares (ECV), así como neoplasias, problemas respiratorios, dermatológicos y ortopédicos resultantes. factores genéticos, ambientales y de estilo de vida inadecuado. Consideraciones finales: La obesidad en la adolescencia es un gran problema de salud pública, y es importante conocer la historia natural de la enfermedad y las comorbilidades para permitir el diagnóstico y tratamiento precoz de estas condiciones.

Palabras clave: Enfermería, Adolescente, Obesidad.

\section{INTRODUÇÃO}

A obesidade é decorrente do acúmulo de gordura no organismo, que está associado a riscos para a saúde devido à sua relação com várias complicações metabólicas. Pode ser compreendida como um agravo de caráter multifatorial, pois suas causas estão relacionadas a questões biológicas, históricas, ecológicas, econômicas, sociais, culturais e políticas (BRASIL, 2017). No Brasil, de acordo com o Ministério da Saúde (2017), as estimativas de prevalência e incidência de obesidade são alarmantes, evidenciadas nas mudanças comportamentais ocorridas nas últimas décadas, em termos de alimentação inadequada e ao sedentarismo cada vez maior da população (VALENTE J, 2018).

Oliveira AMA, et al. (2019) e Viuniski N (2015); informam que existem inúmeros métodos sofisticados de avaliação da obesidade, utilizados por centros médicos ou de pesquisas, sendo um largamente utilizado e recomendado pela Organização Mundial de Saúde (OMS) o Índice de Massa Corporal (IMC), como método válido na verificação do estado nutricional populacional, indicativo de sobrepeso e obesidade. Calculado dividindo-se o peso (em $\mathrm{kg}$ ) pelo quadrado da altura (em metros). O resultado revela se o peso está dentro da faixa ideal, abaixo ou acima do desejado.

O Estatuto da Criança e Adolescente (ECA) (Lei №. 8069/90) em seu art. $2^{\circ}$ dispõe do conceito de adolescente: "Considera-se criança, para os efeitos desta Lei, a pessoa até doze anos de idade incompletos, e adolescentes aquela entre doze e dezoito anos de idade". Porém, os serviços de saúde consideram a adolescência a fase entre 10 e 19 anos (BRASIL, 2009). Carvalho EAA, et al. (2013) afirmam que, atualmente, pode-se dizer que o Brasil vem sofrendo com um aumento expressivo de obesidade em todas as faixas etárias e classes sociais, o que é considerado um grande problema de saúde pública.

Para Lopes M (2011); entre as causas da obesidade, seja em adultos ou adolescentes estão: "escolhas alimentares pobres; má alimentação; uma vida sedentária". Em se tratando de crianças e adolescentes a família exerce papel fundamental, tendo em vista que cabe aos pais a saúde do seu filho, é a sua principal responsabilidade até atingirem idade adulta. Adicionalmente, a equipe de enfermagem poderá estar atuando junto à família em busca de prevenção da obesidade para evitar outras possíveis doenças.

Segundo Silva CM e Polubriaginof C (2012); há diversas complicações que podem estar relacionadas à obesidade, tais como: doenças respiratórias, problemas gastrointestinais, doenças cardiovasculares, câncer e artrite. Para Miranda JMQ, et al. (2011), os adolescentes obesos que permanecem acima do peso podem apresentar complicações neurometabólicas e endócrinas que facilitam o desenvolvimento da doença cardiovascular na idade adulta. Já foi provado que crianças e adolescentes obesos apresentam, antes de atingir a maturidade, fatores de risco para desenvolver doença cardiovascular, hiperinsulinemia, aumento da pressão arterial e disfunção autonômica.

Presume-se que o enfermeiro apto para o cuidado de adolescentes obesos auxiliará identificando os fatores de risco e assim criar métodos de cuidado para esses adolescentes, para prevenir o excesso de peso. Tendo em vista que a família tem grande influência nos hábitos alimentares, os profissionais de saúde também poderão estar promovendo ações de reeducação alimentar. De acordo com Costa NFN, et al. (2016) a educação nutricional, seja em casa, na escola ou em qualquer outro local, pode trazer resultados positivos na redução da obesidade e melhoria da qualidade de vida.

Este artigo trata-se de uma revisão bibliográfica da literatura, que poderá auxiliar os profissionais de saúde a diminuir essa problemática, que é a obesidade na adolescência, intervindo precocemente para evitar complicações na saúde nesta faixa etária. Dessa forma, o objetivo principal deste artigo será analisar evidências científicas relacionadas à obesidade na adolescência. E como objetivos específicos, identificar os principais fatores relacionados com a obesidade e identificar os benefícios dos cuidados de enfermagem na prevenção da obesidade na adolescência. 


\section{MÉTODOS}

Segundo o dicionário definition from the Merriam (2009), ciência ou conhecimento refere-se a qualquer conhecimento ou prática sistemáticas. Em sentido estrito, refere-se ao sistema de adquirir conhecimento baseado no método científico bem como ao conhecimento organizado das pesquisas. Segundo Amaral JF (2007), pesquisas bibliográficas "consistem no levantamento, seleção, fichamento e arquivamento de informações relacionadas à pesquisa". A pesquisa bibliográfica é uma pesquisa que foi feita coletando-se dados de fontes secundárias de informação.

Trata-se de uma Revisão Integrativa da Literatura - RIL, ao qual foi desenvolvida em busca na base de dados LILACS, MEDLINE e BDENF; a seleção dos artigos, de acordo com os critérios de inclusão e exclusão da temática proposta; análise dos artigos, que se enquadram as descrições do tema abordado; ano de publicação, que serão do tipo de estudo e periódico em que foi publicado; construção do artigo e defesa pública do mesmo. Como critérios de inclusão foram utilizados artigos publicados em português, dentro do período delimitado (2013-2019), que retratam a temática em ênfase e com indexação nas bases de dados da Literatura Latino-Americano e do Caribe em Ciências da Saúde (LILACS), Sistema Online de Busca e Análise de Literatura Médica (MEDLINE) e no Banco de Dados de Enfermagem (BDENF). Os critérios de exclusão foram artigos duplicados, com títulos não compatíveis ao tema e os quais não sejam plausíveis ao acesso gratuito ao texto finalizado.

A ferramenta utilizada de busca dos artigos foi a Biblioteca Virtual de Saúde (BVS). Esta busca procedeuse no período de agosto de 2019, a partir do descritor: enfermagem, adolescente e obesidade, ao qual seguidamente procedeu-se à busca na BVS, emergindo 2.003.791 publicações. A partir disso iniciou-se a utilização dos critérios de inclusão com o filtro dos artigos. Primeiro, foi utilizado como filtro o assunto principal: enfermagem com 35.840 resultados, adolescente com 35.297 resultados, e obesidade com 21.369 resultados das 2.003.791 publicações iniciais.

Adicionalmente, foram utilizados como filtro a base de dados: MEDLINE, LILACS e BDENF com 32.719 publicações das 2.003.791 iniciais. A seguir foram utilizados como filtro o limite: humanos, adolescente, feminino e masculino, que emergiu 29.517 publicações, das 2.003.791 iniciais. Seguindo foi feito filtro com o idioma: português, com 3.814 publicações das 2.003.791 iniciais. Após, foi feito o filtro do ano de publicação: 2013 - 2019 emergindo 809 publicações das 2.003.791 iniciais. Seguindo, foi feito o filtro no tipo de documento: artigo com 689 publicações, e finalizando com o filtro do País/Região como assunto: Brasil com 84 publicações.

Após essa seleção, utilizando os critérios de inclusão como filtro na BVS, foi realizada a leitura prévia dos títulos e resumos, sendo selecionados 24 artigos, por suas temáticas serem condizentes ao tema proposto, finalizando com 7 artigos selecionados. A exclusão dos artigos se caracterizou em estudos de artigos repetidos, artigos que não eram plausíveis ao acesso gratuito finalizado e artigos que não se adequaram ao objetivo do tema proposto. Para a coleta de dados, foi utilizado um instrumento que agrega os seguintes aspectos: Título; Descritores; Tipo de Pesquisa; Autores; Ano; Revista; Base de Dados; Objetivo; Resultado; Considerações Finais.

\section{RESULTADOS}

Nesta RIL foram analisados 07 artigos que atenderam aos critérios de inclusão previamente estabelecidos. As publicações dos artigos selecionados foram feitas nos seguintes periódicos: 14,28\% (A2) foi publicado na base de dados MEDLINE; 28,56\% (A1, A3) dos artigos publicados na base de dados da LILACS; 42,84\% (A4, A5, A7) foram publicados na base de dados SCIELO; 14,28\% (A6) foi publicado na base de dados BDENF.

Desses artigos, 14,28\% (A1) foi publicado no ano de 2014, na Revista de Terapia Ocupacional da Universidade de São Paulo; 14,28\% (A2) foi publicado no ano de 2014, na Ciência \& Saúde Coletiva; 14,28\% (A3) foi publicado no ano de 2013 na Ciência \& Saúde Coletiva; 14,28\% (A4) foi publicado no ano de 2019, na Revista Brasileira Epidemiológica; 14,28\% (A5) que foi publicado no ano de 2015, na Ciência \& Saúde Coletiva; $14,28 \%$ (A6) foi publicado no ano de 2017, na Revista Pró-univerSUS; $14,28 \%$ (A7) foi publicado no ano de 2018, na Revista da Escola de Enfermagem da USP. No Quadro 1 estão expostas as informações sobre os artigos selecionados para compor esse estudo. 


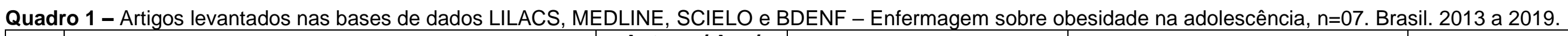

\begin{tabular}{|c|c|c|c|c|c|}
\hline № & Título/ Descritores/ Tipo de Pesquisa & $\begin{array}{c}\text { Autores/Ano/ } \\
\text { Revista/ Base de } \\
\text { Dados }\end{array}$ & Objetivo & Resultado & Considerações Finais \\
\hline A1 & $\begin{array}{l}\text { Desempenho ocupacional de adolescentes escolares } \\
\text { com excesso de peso. } \\
\text { Descritores: Sobrepeso; Obesidade; Adolescente; } \\
\text { Atividades cotidianas; Terapia Ocupacional. } \\
\text { Estudo transversal e descritivo. }\end{array}$ & $\begin{array}{l}\text { MEDEIROS TML, } \\
\text { et al., 2014. Rev. } \\
\text { Ter. Ocup. Univ. } \\
\text { São Paulo. } \\
\text { LILACS }\end{array}$ & $\begin{array}{l}\text { Identificar as atividades- } \\
\text { problema no desempenho } \\
\text { ocupacional de } \\
\text { adolescentes escolares } \\
\text { com sobrepeso/obesidade. }\end{array}$ & $\begin{array}{l}\text { Da amostra inicial de } 86 \\
\text { adolescentes, } 14 \text { estiveram acima } \\
\text { do peso, sendo } 10 \text { com } \\
\text { sobrepeso e } 4 \text { com obesidade. }\end{array}$ & $\begin{array}{c}\text { Foram verificadas } \\
\text { dificuldades no } \\
\text { desempenho ocupacional } \\
\text { dos adolescentes com } \\
\text { sobrepeso/ obesidade, } \\
\text { principalmente nas } \\
\text { atividades produtiva e do } \\
\text { lazer. }\end{array}$ \\
\hline A2 & $\begin{array}{l}\text { Medidas de transmissão intergeracional da obesidade } \\
\text { no Brasil. } \\
\text { Descritores: Obesidade, Mobilidade intergeracional, } \\
\text { Persistência. } \\
\text { As matrizes de transição markovianas e regressões } \\
\text { quantílicas e logísticas. }\end{array}$ & $\begin{array}{l}\text { ALMEIDAATC, et } \\
\text { al., 2014. Ciência } \\
\text { \& Saúde Coletiva. } \\
\text { MEDLINE }\end{array}$ & $\begin{array}{l}\text { Investigar o problema da } \\
\text { obesidade do ponto de } \\
\text { vista intergeracional, a } \\
\text { partir dos índices de massa } \\
\text { corporal (IMC) de pais e } \\
\text { filhos. }\end{array}$ & $\begin{array}{l}\text { Os principais resultados sugerem } \\
\text { a existência de uma forte } \\
\text { associação entre o IMC dos pais } \\
\text { e filhos, com o aumento da fração } \\
\text { de crianças obesas em cenários } \\
\text { familiares monoparentais e em } \\
\text { lares em que ambos os pais são } \\
\text { obesos. }\end{array}$ & $\begin{array}{c}\text { O papel da obesidade } \\
\text { materna sobrepõe-se ao } \\
\text { da paterna em termos de } \\
\text { transmissão } \\
\text { intergeracional da } \\
\text { obesidade para os filhos, } \\
\text { independentemente do } \\
\text { gênero. }\end{array}$ \\
\hline A3 & $\begin{array}{l}\text { Obesidade em adolescentes e as políticas públicas de } \\
\text { nutrição. } \\
\text { Descritores: Políticas de saúde, Adolescentes, } \\
\text { Obesidade } \\
\text { Pesquisa de revisão de literatura. }\end{array}$ & $\begin{array}{l}\text { FREITAS LKP, et } \\
\text { al., 2013. Ciência } \\
\text { \& Saúde Coletiva. } \\
\text { LILACS }\end{array}$ & $\begin{array}{l}\text { Analisar historicamente os } \\
\text { avanços das políticas } \\
\text { públicas no Brasil } \\
\text { relacionadas à } \\
\text { nutrição/alimentação e à } \\
\text { prática de atividade física } \\
\text { no controle da obesidade } \\
\text { em adolescentes. }\end{array}$ & $\begin{array}{l}\text { Os resultados indicaram que as } \\
\text { políticas públicas de nutrição } \\
\text { realizadas no Brasil têm atuado } \\
\text { de forma incipiente quando } \\
\text { destinadas ao adolescente no } \\
\text { que diz respeito ao fator } \\
\text { obesidade. }\end{array}$ & $\begin{array}{l}\text { Faz-se necessário um } \\
\text { olhar mais ampliado em } \\
\text { busca de políticas que } \\
\text { atentem para o controle } \\
\text { da obesidade em } \\
\text { adolescentes. }\end{array}$ \\
\hline A4 & $\begin{array}{l}\text { Prevalência e fatores associados da obesidade na } \\
\text { população brasileira: estudo com dados aferidos da } \\
\text { Pesquisa Nacional de Saúde, 2013. Descritores: } \\
\text { Obesidade. Antropometria. Inquérito de Saúde. Estilo } \\
\text { de vida. Morbidade. Brasil. } \\
\text { Pesquisa de âmbito nacional e base domiciliar } \\
\text { realizada pela Fundação Oswaldo Cruz e pelo } \\
\text { Ministério da Saúde, em parceria com o Instituto } \\
\text { Brasileiro de Geografia e Estatística (IBGE). }\end{array}$ & $\begin{array}{l}\text { FERREIRA APS, } \\
\text { et al., 2019. Rev. } \\
\text { Bras. } \\
\text { Epidemiológica. } \\
\text { SCIELO }\end{array}$ & $\begin{array}{l}\text { Estudar a prevalência e } \\
\text { identificar fatores } \\
\text { associados à obesidade na } \\
\text { população adulta brasileira } \\
\text { com base nos dados } \\
\text { coletados na Pesquisa } \\
\text { Nacional de Saúde, } 2013 \text {. }\end{array}$ & $\begin{array}{l}\text { As prevalências de obesidade } \\
\text { foram de } 16,8 \% \text { para homens e } \\
24,4 \% \text { para mulheres. Idade } \\
\text { avançada (a partir dos } 50 \text { anos). } \\
\text { A atividade física no lazer e o } \\
\text { hábito de assistir mais de } 4 \text { horas } \\
\text { de televisão por dia mostraram } \\
\text { associações significativas para } \\
\text { ambos os sexos. }\end{array}$ & $\begin{array}{l}\text { Os achados enfatizam a } \\
\text { importância de políticas } \\
\text { públicas para a } \\
\text { prevenção da obesidade } \\
\text { e para a promoção de } \\
\text { hábitos saudáveis na } \\
\text { sociedade brasileira. }\end{array}$ \\
\hline
\end{tabular}

REAS/EJCH | Vol.13(1) | e5521 | DOI: https://doi.org/10.25248/reas.e5521.2021 Página 4 de 9 


\begin{tabular}{|c|c|c|c|c|c|}
\hline № & Título/ Descritores/ Tipo de Pesquisa & $\begin{array}{c}\text { Autores/Ano/ } \\
\text { Revista/ Base de } \\
\text { Dados }\end{array}$ & Objetivo & Resultado & Considerações Finais \\
\hline A5 & $\begin{array}{l}\text { Estado nutricional dos alunos da Rede Nacional de } \\
\text { Ensino de Educação Infantil e Fundamental do Serviço } \\
\text { Social do Comércio (Sesc), Brasil, } 2012 . \\
\text { Descritores: Obesidade, Estado nutricional, Pré- } \\
\text { escolar, Crianças, Adolescentes. } \\
\text { Trata-se de um estudo transversal. }\end{array}$ & $\begin{array}{l}\text { ANJOS LA, et al., } \\
\text { 2015. Ciência \& } \\
\text { Saúde Coletiva. } \\
\text { SCIELO }\end{array}$ & $\begin{array}{l}\text { Identificar e descrever o } \\
\text { crescimento e o perfil } \\
\text { nutricional antropométrico } \\
\text { das crianças matriculadas } \\
\text { na Rede Nacional de } \\
\text { Ensino do Sesc. }\end{array}$ & $\begin{array}{l}\text { A prevalência de EMC (excesso } \\
\text { de massa corporal) para crianças } \\
\text { acima de } 10 \text { anos foi de } 37,4 \% \\
\text { ( } 32,5 \% \text { em meninas e } 43,0 \% \text { em } \\
\text { meninos). }\end{array}$ & $\begin{array}{c}\text { As prevalências } \\
\text { encontradas foram } \\
\text { expressivas. Estes } \\
\text { resultados baseados nos } \\
\text { estudos nacionais de alta } \\
\text { prevalência de EMC, em } \\
\text { todas as faixas etárias, } \\
\text { reforçam a necessidade } \\
\text { de acompanhamento do } \\
\text { estado nutricional devido } \\
\text { aos agravos associados } \\
\text { ao EMC infantil e suas } \\
\text { consequências na vida } \\
\text { adulta. }\end{array}$ \\
\hline A6 & $\begin{array}{l}\text { Assistência de enfermagem na prevenção e promoção } \\
\text { da Saúde da criança e adolescente com risco para } \\
\text { obesidade. } \\
\text { Descritores: Enfermagem; Obesidade; Prevenção. } \\
\text { Exploratória descritiva, qualitativa. }\end{array}$ & $\begin{array}{l}\text { PINHO GBB, et } \\
\text { al., 2017. Revista } \\
\text { Pró-univerSUS. } \\
\text { BDENF - } \\
\text { Enfermagem }\end{array}$ & $\begin{array}{l}\text { Identificar os cuidados de } \\
\text { enfermagem na prevenção } \\
\text { e promoção da saúde da } \\
\text { criança e do adolescente } \\
\text { com risco para obesidade. } \\
\text { Caracterizar os cuidados } \\
\text { de enfermagem que } \\
\text { auxiliam na prevenção da } \\
\text { obesidade infantil. }\end{array}$ & $\begin{array}{l}\text { Aplicar na saúde da criança é } \\
\text { pensar em longo prazo, visto que } \\
\text { o adulto é consequência do que } \\
\text { foi quando criança. }\end{array}$ & $\begin{array}{l}\text { A necessidade da } \\
\text { realização da consulta de } \\
\text { enfermagem na } \\
\text { puericultura } \\
\text { principalmente no que se } \\
\text { refere às medidas } \\
\text { antropométricas e seu } \\
\text { acompanhamento e } \\
\text { registro no cartão da } \\
\text { criança. }\end{array}$ \\
\hline A7 & $\begin{array}{l}\text { Programa de Enfermagem Saúde na Escola: } \\
\text { prevenção e controle de sobrepeso/obesidade em } \\
\text { adolescentes. } \\
\text { Descritores: Adolescente; Obesidade; Cuidados de } \\
\text { enfermagem; Atenção Primária à Saúde; Enfermagem } \\
\text { em Cuidados Primários. } \\
\text { Estudo qualitativo. }\end{array}$ & $\begin{array}{l}\text { VIEIRA CENK, et } \\
\text { al., 2018. Rev. } \\
\text { Esc. Enfer. USP. } \\
\text { SCIELO. }\end{array}$ & $\begin{array}{l}\text { Descrever o processo de } \\
\text { construção de uma } \\
\text { intervenção na forma de } \\
\text { um programa de } \\
\text { assistência de enfermagem } \\
\text { a adolescentes, destinado } \\
\text { a prevenir e controlar a } \\
\text { obesidade no ambiente } \\
\text { escolar. }\end{array}$ & $\begin{array}{l}\text { Foi construído o Programa de } \\
\text { Enfermagem em Saúde na } \\
\text { Escola, que deve ser } \\
\text { implementado com } 5 \text { aulas } \\
\text { semanais de exercícios e } 1 \\
\text { sessão semanal de intervenções } \\
\text { de enfermagem no Programa. }\end{array}$ & $\begin{array}{l}\text { A intervenção de } \\
\text { enfermagem orientada } \\
\text { pelo Mapeamento de } \\
\text { Intervenção permite } \\
\text { ações baseadas em } \\
\text { teorias, participação da } \\
\text { comunidade, vínculos } \\
\text { escola / serviço e } \\
\text { continuidade do cuidado. }\end{array}$ \\
\hline
\end{tabular}

Fonte: Guimarães LVS, et al., 2020.

REAS/EJCH | Vol.13(1) | e5521 | DOI: https://doi.org/10.25248/reas.e5521.2021 Página 5 de 9 


\section{DISCUSSÃO}

Os artigos A1, A2, A4 e A6, abordam sobre os hábitos alimentares não saudáveis da família que influenciam na vida de adolescentes sedentários, sendo fatores de risco para a obesidade. Lugão MAS, et al. (2018) consideram que a preferência do consumo de alimentos inadequados, a falta de atividade física, o ambiente familiar e até mesmo influência genética, são fatores que podem desencadear a obesidade infantojuvenil.

Adicionalmente, o estudo A2 aborda sobre a obesidade dos pais ser fator de risco relevante para a obesidade dos filhos, a transmissão intergeracional, relacionada com os hábitos não saudáveis da família, como a má-alimentação e a falta de práticas de atividades físicas. Ademais, os filhos de pais obesos correm um risco duas a três vezes maior de obesidade como adultos em comparação com as crianças de famílias nas quais nenhum dos progenitores é morbidamente obeso. Isso não ocorre apenas por razões genéticas, mas também por causa dos hábitos precários da família em termos de dieta e exercício (MCARDLE WD, et al., 2016).

O estudo A4 relata sobre ter uma alimentação não saudável e exercício físico insuficiente que são os principais fatores de risco para a obesidade. Existem várias evidências sobre a provável presença de mecanismos de regulação do peso corporal que são influenciados por padrões dietéticos inadequados e uma reduzida atividade física em nossa população (FELIPPE F, et al., 2004).

O estudo A6, aborda sobre refletir nos hábitos familiares que podem ser influenciados pelo ambiente escolar no qual o indivíduo está inserido, e a corroboração dos pais em possuir importante participação no programa de intervenção da obesidade, reconhecendo a condição do filho, construindo hábitos alimentares saudáveis e incentivando a prática de exercícios físicos.

A percepção dos pais sobre estado nutricional, imagem corporal e saúde do filho são extremamente importantes, na medida em que os mesmos obtenham conhecimento, para que através da conscientização seja possível iniciar precocemente um tratamento (MACEDO L, et al., 2012).

\section{A influência das redes sociais, jogos eletrônicos e televisão}

Nos artigos A1, A4 e A6, trata-se de haver hábitos de passar de 2 a 4 horas diante de ferramentas tecnológicas, restringindo a prática de atividades físicas. O famoso estilo moderno, no qual a maior parte do tempo livre é passado assistindo televisão, utilizando computadores, jogando vídeo games, é responsável pelo sedentarismo, o que leva ao sobrepeso, obesidade e hipertensão, contribuindo para mortes em faixas etárias cada vez mais jovens (ALVES JGB, et al., 2005).

O artigo A4 retrata sobre a frequência de atividade física, sedentarismo devido as horas assistidas de televisão por dia, são importantes para avaliar o estilo de vida das pessoas. De acordo com a Organização Mundial de Saúde (2003), embora os fatores genéticos predisponham o desenvolvimento da obesidade, os principais determinantes de seu aumento parecem ser os fatores ambientais e comportamentais, como a falta de atividade física, o maior tempo assistindo à televisão e o aumento do consumo de alimentos ricos em açúcares e gorduras.

A mídia utiliza as campanhas publicitárias para a venda de produtos como, sanduiches, refrigerantes, frituras, despertando o interesse e formando hábitos alimentares pouco saudáveis (SERRA GMA e SANTOS $E M, 2017)$. O estudo A6, aborda sobre o consumo alimentar inadequado e falta de práticas físicas, decorrente do uso frequente de computadores, jogos eletrônicos e televisores que influenciam ao estilo de vida do adolescente.

Segundo Damiani D (2018), a etiologia da obesidade é nitidamente multifatorial, caracterizada por um aumento exagerado do consumo de alimentos ricos em gordura e com alto valor calórico, associados ao excessivo sedentarismo condicionado por redução na prática de atividades físicas e incremento de hábitos que não geram gasto calórico, como o ato de assistir TV, o uso prolongado de vídeo games e computadores, entre outros. Enfim, faz-se necessária uma séria mudança no estilo de vida. 


\section{Doenças relacionadas à obesidade}

Os artigos A3, A4, A6 e A7, abordam sobre a problemática que a obesidade acarreta na adolescência, tornando-se um problema de saúde pública. O estudo $A 3$, refere sobre a obesidade estar relacionada aos riscos de doenças associadas, tais como diabetes e doenças cardiovasculares (DCV), bem como, outras alterações, como neoplasias, problemas respiratórios, dermatológicos e ortopédicos decorrentes de fatores genéticos, ambientais e de estilo de vida inadequado. Sendo, muito relevante e importante o conhecimento dessas comorbidades para permitir o diagnóstico precoce e o tratamento destas condições.

Para Salve MGC (2006), a obesidade tem como causas as alterações hormonais, genéticas, socioculturais, psicológicas, diminuição do gasto energético, aumento da ingestão alimentar e sedentarismo. A obesidade pode acarretar elevação dos triglicerídeos e colesterol, alterações ortopédicas, problemas respiratórios, diabetes mellitus, hipertensão arterial, entre outros distúrbios. Além disso, uma criança obesa aumenta a probabilidade de se tornar um adulto obeso, o que pode gerar uma gama de problemas de saúde, tendo como consequência até a diminuição da expectativa de vida (CORSO ACT, et al., 2003).

$\mathrm{O}$ artigo A4, destaca a obesidade como fator de risco para uma série de doenças, sendo o obeso mais propenso a desenvolver problemas como: hipertensão, doenças cardiovasculares, diabetes tipo 2, além de problemas físicos como artrose, pedra na vesícula, artrite, cansaço, refluxo esofágico, tumores de intestino e de vesícula, dentre outras de acordo com Bahia L, et al. (2012).

Segundo Iser BPM, et al. (2016) no Brasil, a prevalência da obesidade é muito elevada provocando graves problemas de saúde e de riscos para doenças cardiovasculares, metabólicas, neoplásicas, ortopédicas, entre outras. Por ser de causa multifatorial resultante da interação de fatores genéticos, metabólicos, sociais, comportamentais e culturais, causa forte impacto tanto na saúde quanto no bem-estar psicológico e, principalmente, na qualidade de vida (BORGES HP, et al., 2008; BAHIA L, et al., 2012; OYEBODE O, et al., 2015).

O A6, aborda sobre os diversos fatores que podem desencadear a obesidade. Entre eles os aspectos: genéticos, ambientais, sociais e econômicos. Como alteração endócrina e metabólica, além de acarretar problemas psíquicos e hormonais. Segundo Prati SORA e Petroski EL (2001); problemas de ordem psicológica podem ser apresentados por pessoas portadoras de obesidade, principalmente com relação à autoestima que, por sua vez, pode limitar as relações interpessoais, além de aumentar as dificuldades na prática de atividades físicas.

Vários fatores são importantes na gênese da obesidade, como os genéticos, os fisiológicos e os metabólicos. No entanto, os que poderiam explicar este crescente aumento do número de indivíduos obesos parecem estar mais relacionados às mudanças no estilo de vida e aos hábitos alimentares. Confirmando a influência do meio ambiente sobre o desenvolvimento do excesso de peso em nosso meio e a influência do fator socioeconômico e do micro-ambiente familiar (OLIVEIRA AMA, et al., 2019).

O estudo A7, aborda sobre os adolescentes que apresentam a obesidade, que estão em maior risco de desenvolver problemas de saúde graves, tais como diabetes, doenças cardíacas, hepáticas, respiratórias e psicológicas. Segundo Vilela ALM (2011); ocasiona sérios problemas psicológicos, acarretando também frustrações, infelicidade, além de uma gama enorme de doenças lesivas. O aumento da obesidade tem relação com: o sedentarismo, a disponibilidade atual de alimentos, erros alimentares e pelo próprio ritmo desenfreado da vida atual.

\section{Prevenção da obesidade}

Os artigos A3, A4, A5, A6 e A7 abordam sobre prevenção da obesidade que deve ser de maneira sistemática e multifatorial, impedindo a progressão da doença para um estágio mais grave e consequentemente prevenindo as complicações. Associada sobretudo a hábitos de vida sedentários e a uma alimentação inadequada, a obesidade é, no entanto, uma doença de fisiopatologia complexa e de tratamento difícil. Para que seja eficaz, deve ser um tratamento a longo prazo e manter constante vigilância na prática regular de atividade física e de controle da ingestão calórica, além de outros fatores, como apoio social, familiar e intervenções de saúde na sua prevenção, principalmente em crianças e adolescentes. 
Pesquisas revelam que a Educação Alimentar e Nutricional (EAN), como um modelo de intervenção, tem sido uma estratégia promissora nas escolas com ampliação dos conhecimentos e das atitudes em torno da alimentação, assim como nas práticas e no comportamento alimentar infanto-juvenil, influenciando também os hábitos alimentares de toda a família (TRICHES RM e GIUGLIANIB ERJ, 2005).

Fernandes RA e Vargas AS (2007), dizem que a família pode e deve contribuir com práticas que visem prevenir a obesidade infanto-juvenil da seguinte forma: Com folders educativos, vídeos, relatos, de forma a conscientizá-los acerca das causas e consequências do peso em excesso, orientá-los quanto à necessidade da fixação de horários para alimentar-se, cardápios que condizem com a oferta de boa alimentação, direcionar ações que visem promover saúde e melhor qualidade de vida, fazem parte das atribuições do enfermeiro junto ao meio familiar.

O estudo A4, demostra o perfil da obesidade na população brasileira com ritmo aumentado preocupante, sendo de grande relevância esses estudos para se entender os padrões de riscos e os fatores associados, subsidiando políticas públicas de prevenção da obesidade e para a promoção de hábitos saudáveis na sociedade brasileira. Diante das projeções de prevalência da obesidade, demarca-se o nível de Atenção Primária à Saúde (APS), que tem papel fundamental na gestão do cuidado das pessoas do território adstrito com a atuação de suas equipes multidisciplinares e, em especial, o papel do enfermeiro (BRASIL, 2012).

As pessoas inativas devem iniciar com atividades de curta duração e baixa intensidade, progredindo até volumes mais adequados, como atividades moderadas: caminhar, dançar ou realizar atividades domésticas, ou mesmo as atividades vigorosas que incluem a corrida, pedalar ou nadar em ritmo forte, mover ou levantar cargas pesadas. Ressalte-se que o gasto calórico durante as atividades físicas varia com a dosagem (intensidade e duração) do esforço e o peso do indivíduo. Além disso, a atividade física deve ser prazerosa, de preferência ao ar livre e na companhia de amigos ou familiares (NAHAS MV, 2013).

$\mathrm{O}$ artigo A5, evidencia a complexidade e o elevado custo para o tratamento da obesidade, constituindo como grande desafio sua prevenção no quadro epidemiológico nacional. Adicionalmente, a identificação precoce do estado nutricional dos grupos mais vulneráveis demostra um melhor direcionamento das políticas de saúde pública para minimizar os riscos de doenças crônicas não transmissíveis ligadas à alimentação e ao estilo de vida na vida adulta.

Os profissionais de saúde, em especial a enfermagem, devem ser capazes de reconhecer nos discursos desta clientela informações que possibilitarão o planejamento de ações concretas, individuais e coletivas que sejam mais efetivas no controle deste agravo que, consequentemente, causarão uma melhora na qualidade de vida nesta fase e minimizarão a chance de doenças crônicas e suas complicações na vida adulta (VICTORINO SVZ, et al., 2014).

Ações conjuntas e sistemáticas entre os profissionais da saúde e os da educação, envolvendo as famílias e a comunidade, auxiliam na confrontação da obesidade infantil, promovendo melhora na qualidade de vida da população (SANTOS FDR, et al., 2014). O estudo A6, O enfermeiro gera ações de prevenção primária no controle da obesidade através de ações educativas. Instruindo acerca das dietas alimentares e da prática de atividades físicas, encorajando e apoiando os pacientes por meio de ações educativas, durante todo o período de prática das ações de prevenção e promoção dos riscos a obesidade.

Nesse contexto, é fundamental que o enfermeiro enquanto educador participe diretamente da prevenção e tratamento da obesidade, criando protocolos de enfermagem (RIBEIRO KRA, et al., 2015). Para que haja adoção de condutas de prevenção, controle e tratamento há a necessidade de maior compreensão de aspectos relacionados a esta patologia e suas complicações por parte dos responsáveis diretos pela população infanto-juvenil, do núcleo escolar e dos profissionais de saúde (MATOS JC, et al., 2015).

O estudo A7, aborda sobre a prática de enfermagem, onde a participação ativa da comunidade poderia alcançar melhores resultados na prevenção e no controle desse problema de saúde. O processo de construção de uma intervenção em forma de programa para assistência de enfermagem ao adolescente, direcionado à prevenção e ao controle de sobrepeso/obesidade na escola, inserido através da necessidade da "disponibilização de exercícios físicos na escola", o que pode aumentar a autoestima, a aceitação social e a sensação de bem-estar, além de auxiliar na prevenção de doenças cardiovasculares e mentais crônicas. 
$\mathrm{Na}$ escola a obesidade reflete nas crianças atingidas, promovendo um desconforto, limitando a capacidade das mesmas de realizar atividades físicas delegadas pelos professores (RIBEIRO KRA, et al., 2015). Ressalta-se a importância da atuação de profissionais de saúde, como os enfermeiros, em escolas, onde se têm a oportunidade de realizar diversas ações, de forma contínua e incessante junto às crianças e todos os envolvidos, para a detecção de problemas de saúde e ações de promoção (SOUZA MHN, et al., 2013).

\section{CONSIDERAÇÕES FINAIS}

Espera-se que as referências oferecidas neste artigo, disponibilize o conhecimento da patologia e impulsione os enfermeiros e acadêmicos de enfermagem a buscar técnicas de abordagem e identificação precoce da doença, para que assim seja possível oferecer um cuidado para esses adolescentes obesos, e possivelmente contribuir com profissionais de saúde no desenvolvimento de ações que venham diminuir a incidência da obesidade e colocar em práticas as condutas educativas em relação as adaptações de um novo estilo de vida saudável.

\section{REFERÊNCIAS}

1. ALVES JGB, et al. Prática de esportes durante a adolescência e atividade física de lazer na vida adulta. Rev. Bras. Med. Esporte, 2005; $11(5): 291-4$

2. AMARAL JF. Como fazer uma pesquisa bibliográfica. Universidade Federal do Ceará, 2007. $21 \mathrm{p}$.

3. BAHIA L, et al. The costs of overweight and obesity-related diseases in the Brazilian public health system: cross-sectional study. BMC Public Health, 2012; 12: 440.

4. BORGES HP, et al. Associação entre Hipertensão Arterial e Excesso de Peso em Adultos em Belém, Pará. Arq Bras Cardiol, 2008; 91(2): $110-8$

5. BRASIL. Ministério da Saúde. Secretaria de Atenção à Saúde. Departamento de Atenção Básica. Saúde na escola/Ministério da Saúde, Secretaria de Atenção à Saúde, Departamento de Atenção Básica. Brasília: Ministério da Saúde, 2009.

6. BRASIL. Ministério da Saúde.Secretaria de Atenção à Saúde. Departamento de Atenção Básica.Política Nacional de Atenção Básica. - Brasília: Editora do Ministério da Saúde, 2012.

7. CARVALHO EAA, et al. Obesidade: Aspectos epidemiológicos e prevenção.Rev. Med., 2013; 23(1): 74-82

8. CORSO ACT, at al. Sobrepeso em crianças menores de 6 anos de idade em florianópolis, SC. Revista de Nutrição, 2003; 16(1).

9. COSTA NFN, et al. A influência das novas tecnologias no sedentarismo atual. Anais do XXII Encontro dos Estudantes de Educação Física, 2016; 1(3):1-11.

10. DAMIANI D. Obesidade na infância e adolescência: um extraordinário desafio! Arquivo Brasileiro de Endocrinologia \& Metabologia, 2018; 44(5):363-365.

11. FELIPPE F, et al. Obesidade e a mídia: o lado sutil da informação. Rev Acadêmica do Grupo Comunicacional de São Bernardo, $2004 ; 1(2)$.

12. FERNANDES RA e VARGAS SA. O cuidado de enfermagem na obesidade infantil. Revista Meio Ambiente e Saúde, 2007; 2(1)

13. ISER BPM, et al. Trends in the prevalence of self-reported diabetes in Brazilian capital cities and the Federal District, $2006-2014$. DiabetolMetabSyndr, 2016; 8(70).

14. LOPES M. Causas da Obesidade Infantil - Crianças Obesas como evitar em casa. 2011

15. LUGÃO MAS, et al. A importância da atuação do enfermeiro na prevenção da obesidade infantil. Rev. Cuidado Fundamental Online, 2018; 2(3):976-988.

16. MACEDO L, at al. Percepções parentais sobre estado nutricional, imagem corporal e saúde em crianças com idade escolar. Rev. Enf. Ref., 2012; 3(6):191-200

17. MATOS JC, et al. Atuação do enfermeiro na prevenção da obesidade infantil em uma capital do nordeste. Revista Eletrônica Gestão \& Saúde, 2015; 6(3):2608-22.

18. MCARDLE WD, et al., Energia, Nutrição e Desempenho Humano. Fisiologia do Exercício. Rio de Janeiro: Guanabara, 2016.

19. MINISTERIO DA SAÚDE. Atenção Éspecializada e Hospitalar. Obesidade. Ministério da Saúde: 2017.

20. SCIENCE. 2009. In: Definition from the Merriam. Merriam-Webster Online Dictionary.

21. MIRANDA JMQ, et al. Obesidade infantil e fatores de risco cardiovasculares. ConScientiae Saúde, 2011; 10(1): 175-180.

22. NAHAS MV. Atividade física, saúde e qualidade de vida. 6. ed. Londrina: Midiograf, 2013.

23. OLIVEIRA AMA, et al. Influência de fatores biológicos e ambientais em Feira de Santana, BA. Arquivos Brasileiros de Endocrinologia e Metabologia, 2019; 47(2): 23-45.

24. ORGANIZAÇÂO MUNDIAL DE SAÚDE. Global strategy on diet, physical activity and health: World Health Organization. Genebra: WHO; 2003.

25. OYEBODE $O$, et al. Fruit and vegetable consumption and all-cause, cancer and CVD mortality: analysis of Health Survey for England data.J Epidemiol Community Health, 2015; 68(9): 856-62.

26. PRATI SORA, PETROSKI EL. Atividade física em adolescentes obesos. Revista de Educação Física, 2001, 12(1):59-67.

27. RIBEIRO KRA, et al. Ação da enfermagem no combate à obesidade infantil no período escolar. Revista Recien, 2015; 5(15):11-15

28. SALVE MGC. Obesidade e peso corporal: riscos e consequências. Movimento e Percepção, 2006; 6(8):29-48.

29. SANTOS FDR, et al. Ações de enfermeiros e professores na prevenção e no combate à obesidade infantil. Rev Rene, 2014;15(3):463-70.

30. SERRA GMA, SANTOS EM. Saúde e mídia na construção da obesidade e do corpo perfeito. Ciênc. Saúde Coletiva, 2017; 8(3): 691-701.

31. SILVA CM, POLUBRIAGINOF C. Obesidade infantil: Fatores de risco e intervenções de enfermagem pertinentes. Rev. Enferm. UNISA, 2012; 13(2):112-116.

32. SOUZA MHN, et al. Avaliação do estado nutricional e da saúde de crianças e adolescentes na prática assistencial do enfermeiro. Cogitare Enferm. Curitiba, 2013; v18(1):29-35.

33. TRICHES RM, GIUGLIANIB ERJ. Obesidade, práticas alimentares e conhecimentos de nutrição em escolares. Revista de Saúde Pública, 2005;39(4):541-547.

34. VALENTE, J. Repórter da Agência Brasil. Obesidade atinge quase $20 \%$ da população brasileira, mostra pesquisa.

35. VICTORINO SVZ, et al. Viver com obesidade infantil: a experiência de crianças inscritas em programa de acompanhamento multidisciplinar. Revista Rene, 2014;15(6):980-9.

36. VILELAALM. Obesidade. 2011.

37. VIUNISKI N. ABESO, Associação Brasileira para o Estudo da Obesidade e Síndrome Metabólica. Pontos de Corte de IMC Para Sobrepeso e Obesidade em Crianças e adolescentes. 2015. 